Research Article

\title{
Gelatinases Expression Disturbance as a Possible Cause of Fibromuscular Dysplasia of Internal Carotid Arteries: Immunohistochemical Study
}

\author{
Ekaterina M. Paltseva ${ }^{1,}$, Viktoria O. Polyakova ${ }^{2}$, Svetlana A. Oskolkova ${ }^{3}$, Arsen V. Abramyan ${ }^{1}$, \\ Julia S. Krylova ${ }^{2}$, Alexandre V. Gavrilenko ${ }^{1}$, Igor M. Kvetnoy ${ }^{2}$ \\ ${ }^{1}$ B. V. Petrovsky Russian Research Center of Surgery, Moscow, Russia \\ ${ }^{2}$ D. O. Ott Research Institute of Obstetrics, Gynecology and Reproductology, Saint Petersburg, Russia \\ ${ }^{3}$ I. M. Sechenov First Moscow State Medical University, Moscow, Russia
}

\section{Email address:}

paltseva-k@yandex.ru (E. M. Paltseva)

*Corresponding author

\section{To cite this article:}

Ekaterina M. Paltseva, Viktoria O. Polyakova, Svetlana A. Oskolkova, Arsen V. Abramyan, Julia S. Krylova, Alexandre V. Gavrilenko, Igor M. Kvetnoy. Gelatinases Expression Disturbance as a Possible Cause of Fibromuscular Dysplasia of Internal Carotid Arteries: Immunohistochemical Study. International Journal of Clinical and Experimental Medical Sciences. Vol. 2, No. 4, 2016 , pp. 52-58. doi: 10.11648/j.ijcems.20160204.11

Received: March 16, 2016; Accepted: May 17, 2016; Published: July 5, 2016

\begin{abstract}
Background: Fibromascular dysplasia of internal carotid arteries (ICA) leading to their pathological deformities is one of the causes of cerebral vascular insufficiency. The structural changes of the artery wall and their causes remain poorly understood. Materials and Methods: We investigated the expression of elastin, collagen types I and III, smooth muscle cells, gelatinases degrading elastin (matrix metalloproteinases 2 and 9 (MMP2 and MMP9) and tissue inhibitors of matrix metalloproteinases 1 and 2 (TIMP1 and TIMP2) on formalin-fixed surgical samples with the methods of immunohistochemistry and confocal laser scanning microscopy. Results: We revealed the fragmentation of elastic fibers (100\% of patients) and some reduction of smooth muscle cells $(\mathrm{p}<0.05)$ in the tunica media of ICA. There were no changes in collagen types I and III and TIMP2 expression. The study of the ratio of the expression of MMPs and TIMPs revealed the statistically significant predominance of high MMP2 and -9 and low TIMP1 content in ICA with pathological deformities. With the use of confocal microscopy, we showed the decrease of elastin expression with a high MMP9 activity which correlated with low expression of TIMP-1 in the group of ICA with pathological deformities. While in the control group there was a high level of elastin expression and a low level of MMP9 expression that correlated with the low TIMP-1 amount $(\mathrm{p}<0.05)$. Conclusion: Our data demonstrate that the main feature of fibromuscular dysplasia underlying the pathological deformities of ICA-fragmentation of elastic fibers is caused by the disturbance of balance between gelatinases and their inhibitors.
\end{abstract}

Keywords: Pathological Deformities of Internal Carotid Artery, Elastin, Collagen, Smooth Muscle Cells, Matrix Metalloproteinase, Tissue Inhibitor of Matrix Metalloproteinases

\section{Introduction}

Among the causes of cerebral vascular insufficiency one of the most important is fibromascular dysplasia of internal carotid arteries (ICA) leading to their pathological deformities. ${ }^{1}$ Pathological deformities of ICA occur at 5-7\% of the adult population..$^{2-4}$ Most investigators suppose that this is the result of congenital ${ }^{5,6}$ or acquired factors (arterial hypertension, atherosclerosis, ageing process), ${ }^{7-9}$ but the etiology of such changes is still not completely understood.

The most common classification of pathological deformities of ICA is that suggested by Weibel J. and Fields W. in $1965 .^{10,11}$ The authors distinguished 3 types of deformities: tortuosity, coiling and kinking. Coiling is characterized by 
congenital circular deformation with the formation of the loop, which may result in cerebral vascular insufficiency. Tortuosity is S- or C-deformation of ICA without formation of sharp angles. Kinking constitutes acquired hemodynamically significant curved ICA (the angle of bending is sharp) with stenosis of its lumen. At the base of configuration anomalies lies the changing of histological patterns. The last two types develop due to atherosclerosis or fibromascular dysplasia of the artery wall. ${ }^{12}$

In pathological deformities caused by fibromascular dysplasia, which is a noninflammatory vascular disease of arteries of small and medium caliber occurring most commonly in renal and carotid arteries, the changes involve all the layers of the artery wall. In tunica intima there is an irregular hyperplasia, in tunica media - the fragmentation of elastic fibers, the formation of fibrous tissue, degeneration of collagen fibers and smooth muscle cells. ${ }^{13}, 14$ The leading morphological sign of fibromuscular dysplasia of ICA is the disruption of the elastic fiber formation: uneven thickening, fragmentation, splitting and the presence of immature elastic fibers. Along with this the improper formation of smooth muscle structures in tunica media is distinctive: chaotic orientation of smooth muscle cells, their hypertrophy and atrophy, progressive reduction in the number, the signs of functional activity of their ultrastructures. ${ }^{15}$ In tunica adventitia there is the fibrosis, mostly pronounced along the small curvature of ICA which fixes the artery in a curved position. ${ }^{13,14}$

The elastic and collagen fibers play the central role in determining the mechanical properties of the vessel wall ensuring its tensile strength. This is achieved by the particular structure of the middle layer which is primarily composed of circumferentially aligned smooth muscle cells and elastin, which is arranged in fenestrated lamella into a three dimensional continuous network between collagen fibers and thin layers of proteoglycan-rich ECM. The relatively high content of collagen fibers, primarily collagen types I and III, in the adventitia helps to prevent vascular rupture at high pressures. The amount of collagen determines the tensile strength of the artery. The increase of the stiffness of the vessel determined by reduction of elastin content and enhancement of collagen takes place under different pathological processes. The low amount of elastin leads to smooth muscle proliferation, reduction of the vessel lumen and stenosis. The poor maintenance of collagen type III results in the fragility of the vessels and the predisposition to rupture of large arteries. Smooth muscle cells perform the contractile and synthetic functions, producing collagens and elastin, cytokines and proteases. The alteration of their amount and prevailing of synthetic function over contractile lead to the disturbance of normal ratio between the ECM components. ${ }^{16}$

Any alteration of ECM structure means the failure of stable balance between the intensity of protein synthesis and degradation. Elastin, the fragmentation of which is the most important histological change in fibromascular dysplasia of ICA, is degraded by MMP 2 and 9. It is well known that MMP 2 and 9 play a significant role in the pathogenesis of cardiovascular diseases. In the formation of aneurysms of the aorta the loss of elastin and collagens has the key role, the reason of which is the enhancement of production of different proteases including MMP1, -2 and $-9 .{ }^{17,}{ }^{18}$ Meanwhile the TIMP1 and TIMP2 expression is lowered that leads to the degradation of ECM components of the aneurismal aortic wall. ${ }^{19,20}$ The balance between the activity of MMPs and their inhibitors is the necessary condition of normal physiological processes.

Despite the active research of clinical implications of fibromuscular dysplasia of ICA nowadays the changes of structure of the artery wall remain poorly understood. The aim of the current study was to investigate the expression of the main components of tunica media - elastin, collagen types I and III, smooth muscle cells. Due to the fact that fragmentation of elastin is the main histological feature of fibromuscular dysplasia underlying the pathological deformities of ICA, gelatinases degrading elastin (MMP2 and MMP9) and their inhibitors TIMP1 and TIMP2 were explored as well.

\section{Materials and Methods}

\subsection{Patients and Tissue Samples}

Among ICA specimens of patients who received surgical treatment for the hemodynamically significant pathological deformities we have chosen those with fibromuscular dysplasia of vascular wall, proven by morphological examination of formalin-fixed paraffin-embedded material $(n=32,6$ men, 26 women, middle age 57,06 $\pm 9,45)$. For histological analysis the samples were stained using the standard hematoxylin-eosin staining technique. 11 specimens of internal mammary arteries, obtained at mammarocoronary bypass surgery, constituted the control group ( 7 men, 4 women, middle age $59,27 \pm 8,27)$. Depending on the type of deformation the patients were divided as follows: with coiling $-5(16 \%)$ patients, with kinking $-8(25 \%)$, with C-shape deformation - $3(9 \%)$ and S-shape - $16(50 \%)$ patients. Patients were divided into three groups according to age classification by the World Health Organization: 1 - young people ( $<44$ years), $2-$ middle age (45-59 years) and $3-$ elderly people (60-74 years). Informed consent was given by each patient before each investigation and the study was approved by the Local Committee of Medical and Biological Ethics of B.V. Petrovsky Russian Research Center of Surgery, Ethics Committee of D.O. Ott Research Institute of Obstetrics, Gynecology and Reproductology, and Local Ethics Committee of I.M. Sechenov First Moscow State Medical University.

\subsection{Immunohistochemistry and Evaluation}

3-4 micrometer thick sections were deparaffinized step-wise in xylene and rehydrated in graded solutions of ethanol. Antigen retrieval and immunoperoxidase staining was performed in fully automated immunohistostainer BOND-MAX (Leica Biosystems, Germany). Bond Polymer 
Refine Detection system (Leica Biosystems, Germany) was used and contained a peroxide block (the incubation time 5 $\mathrm{min}$ ), post primary (the incubation time $8 \mathrm{~min}$ ), polymer reagent $(8 \mathrm{~min})$, DAB chromogen $(10 \mathrm{~min})$ and hematoxylin counterstain. The sections were incubated with primary antibodies after blocking of the non-specific sites for $15 \mathrm{~min}$ at room temperature. Mouse monoclonal primary antibodies were obtained from Abcam (UK; to elastin, clone 10B8, dilution 1:400; collagen I, clone 3G3, dilution 1:200; collagen III, clone Col-29, dilution 1:100; MMP2, clone 6E3F8, dilution 1:200; MMP9, clone 5G3, dilution 1:200; TIMP2, clone 3A4, dilution 1:50) and Dako (Denmark; Actin (Smooth Muscle), clone 1A4, dilution 1:400; TIMP1, clone VT7, dilution 1:100). After staining with hematoxylin sections were dehydrated stepwise through a graded alcohol series, and cleared in xylene before mounting. Consecutive sections from each sample processed without the primary antibody were used as negative control. For positive controls we used following tissues in accordance with the recommendations given in the instructions for the primary antibodies: skin for elastin, collagen I and III, normal uterine myometrium for SMA, ovary tissue for MMP2, brain tissue for MMP9, breast carcinoma for TIMP1 and colon carcinoma - for TIMP2.

All analyses of immunohistochemically stained sections were performed using light microscopy. Elastin, collagens, MMPs and TIMPs immunopositivity was evaluated in the extracellular matrix. SMA expression was observed in the cytoplasm of smooth muscle cells, which were counted according to the previously proposed method in 5 high power fields (x400) in each sample [15]. Expression levels of all other proteins were assessed semiquantitatively: "-" - the absence of expression, "+" - low expression; "++" moderate and " +++ " - high expression. For the analyses of obtaining data the types of expression were united in 2 groups: low and high. Images were captured using a digital camera (Leica Biosystems, Germany).

\subsection{Confocal Laser Scanning Microscopy}

To determine the ratio of the desired markers, we used the following combinations of primary antibody for each sectionMMP9/Elastin and MMP9/TIMP-1. The study was carried out on thin deparaffinized and dehydrated sections (2-4 microns). The temperature antigen unmasking was performed using $0.01 \mathrm{M}$ citrate buffer $(\mathrm{pH}=6.0)$ under pressure. Phosphate buffered saline with Tween-20 was used to wash sections. Sections were incubated for 30 min with blocking serum at room temperature. After washing primary antibody was applied first to the MMP-9 (clone 439, Novocastra, 1:80) and incubated for $1 \mathrm{~h}$ at room temperature. As secondary antibody Alexa fluor $647^{\circledR}$ (Abcam) was used. After further washing the sections were incubated with a second primary antibody to Elastin (clone 10V8, Abcam, 1:400) or to TIMP-1 (clone VT1, Dako, 1:100) for $1 \mathrm{~h}$ at room temperature. As secondary antibody Alexa fluor $488^{\circledR}$ (Abcam) was used. After washing the sections additionally stained with DAPI (AppliChem). All sections were placed in mounts Wednesday (Dako). After procedures above the color results were as following: first antibodies - red fluorescence; second antibodies - green fluorescence; double staining - blue fluorescence; contrasted core - blue fluorescence. The study was carried out on the confocal laser scanning microscope Olympus FV1000D. To study the expression of signaling molecules the spectra and fluorescence quantified characteristics were obtained. We measured two parameters - the relative area of expression and area of co-localization. The relative area of expression calculated as the ratio of the area occupied by immunopositive cells to the total area of cells in the visual field and expressed as a percentage. The fluorescence of certain spectral characteristics was registered only in a plane (2D).

\subsection{Statistical Analyses}

The statistical analyses of our data were performed on a personal computer using software «SPSS Statistics 17.0 for Windows». The age of patients and results of confocal laser scanning microscopy research are presented as mean \pm standard deviation. The significance of differences between the compared groups was determined using the Chi square test and the exact method of Fisher. For all statistical tests the differences were defined as significant at $\mathrm{p}<0.05$.

\section{Results}

\subsection{Immunohistochemical Research}

\subsubsection{Structure of Internal Carotid Artery Wall in Pathological Deformities}

The histological research of ICA with fibromuscular dysplasia revealed the thickened tunica intima, the impairment of the structure of tunica media characterized by elastic fiber fragmentation, irregular collagen fiber arrangement, the formation of fibrous areas, in some places the internal elastic membrane destruction was noted (Figure 1a).

Immunohistochemically the expression of elastin was higher in the tunica media of ICA with pathological deformities than in the control group $(\mathrm{p}<0.05$; Table 1$)$ but the elastic fibers were fragmented (Figure 1b). The collagen I expression was similar in both groups: it was low in most cases (91\% in each group; Figure 1c). We did not observe a difference by comparing the collagen III - the low expression prevailed in both groups (30 out of 32 patients with ICA deformities and 8 out of 11 patients of the control group; Table 1, Figure 1d, e). Meanwhile the expression of both types of collagens in adventitia was high in two groups.

The comparison of SMA revealed the significant differences between our groups $(\mathrm{p}<0.05)$ : its expression was high in $63 \%$ (20 out of 32 ) and low in $37 \%$ (12 out of 32 ) cases of pathological deformities of ICA, whereas in the control group its high expression was detected in all cases $(100 \%)$ (Table 1, Figure 1f).

We did not find any correlations between the degree of expression of elastin, collagens I and III, SMA and gender and age, neither in group of pathological deformities of ICA, neither in the control group $(p>0.05)$. There were no associations of these 
markers with types of pathological deformities $(\mathrm{p}>0.05)$.

Table 1. Expression of elastin, collagens I and III, and SMA in internal carotid artery (ICA) with pathological deformities and in control group.

\begin{tabular}{|c|c|c|}
\hline Expression of markers & ICA with pathological deformities, $n=32$; absolute number (\%) & Control group, $\mathrm{n}=11$; absolute number $(\%)$ \\
\hline \multicolumn{3}{|l|}{ Elastin } \\
\hline Low & $6(19)$ & $7(63.5)$ \\
\hline High & $26(81)^{*}$ & $4(36.5)$ \\
\hline \multicolumn{3}{|l|}{ Collagen I } \\
\hline Low & $29(91)$ & $10(91)$ \\
\hline High & $3(9)$ & $1(9)$ \\
\hline \multicolumn{3}{|l|}{ Collagen III } \\
\hline Low & $30(94)$ & $8(73)$ \\
\hline High & $2(6)$ & $3(27)$ \\
\hline \multicolumn{3}{|l|}{ SMA } \\
\hline Low & $12(37)^{*}$ & 0 \\
\hline High & $20(63)$ & $11(100)$ \\
\hline \multicolumn{3}{|l|}{ MMP2 } \\
\hline Low & $4(13)$ & $4(36.5)$ \\
\hline High & $28(87)$ & $7(63.5)$ \\
\hline \multicolumn{3}{|l|}{ MMP9 } \\
\hline Low & $5(16)$ & 0 \\
\hline High & $27(84)$ & $11(100)$ \\
\hline \multicolumn{3}{|l|}{ TIMP1 } \\
\hline Low & $27(84)^{*}$ & $2(18)$ \\
\hline High & $5(16)$ & $9(82)$ \\
\hline \multicolumn{3}{|l|}{ TIMP2 } \\
\hline Low & $7(22)$ & $3(27)$ \\
\hline High & $25(78)$ & $8(73)$ \\
\hline
\end{tabular}

SMA: smooth muscle actin, MMP2: matrix metalloproteinase 2, MMP9: matrix metalloproteinase 9, TIMP1: tissue inhibitor of matrix metalloproteinases type 1, TIMP2: tissue inhibitor of matrix metalloproteinases type $2,{ }^{*}-\mathrm{p}<0.05$

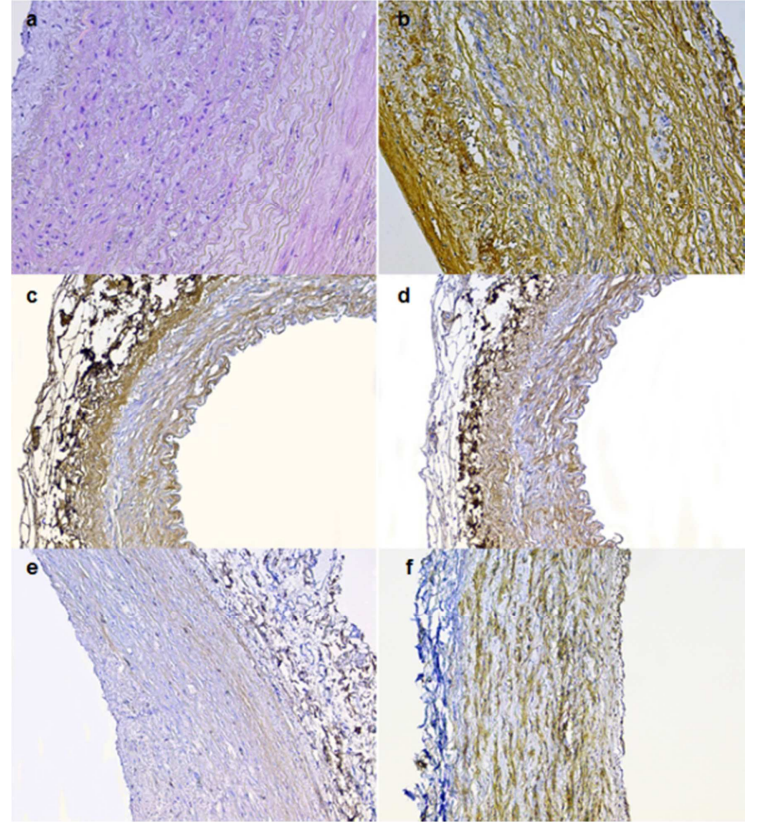

Figure 1. a-Fibromuscular dysplasia of internal carotid artery (ICA). The thickened tunica intima, elastic fiber fragmentation and formation of fibrous areas in tunica media, internal elastic membrane destruction can be noted (x200 magnification, hematoxylin-eosin staining). b- $f$ - Elastin, collagen types $I$ and III, smooth muscle actin expression evaluated by immunohistochemistry. $b$ - high expression of elastin in ICA with fibromuscular dysplasia, but elastic fibers are fragmented (x200 magnification). Collagen types I (c) and III (d) expression in normal artery (x100 magnification). Decreased expression of collagen III (e) and high expression of smooth muscle actin (f) in ICA with fibromuscular dysplasia (x200 magnification).

\subsubsection{Expression of MMP2 and MMP9 and Their Inhibitors TIMP1 and TIMP2}

Immunohistochemical research of MMP2 did not show a statistically significant difference between 2 groups, but there were more cases with its high amount in tunica media among patients with pathological deformities of ICA than in control group ( 87 and $63.5 \%$ respectively, $p=0.17$; Figure 2a). The comparison of MMP9 expression demonstrated that in group of ICA with pathological deformities, there was its high expression in $84 \%$ ( 27 out of 32 ) and low - in $16 \%$ (5 out of 32 ) of cases while in control group its high expression was indicated in all cases (100\%; Figure $2 \mathrm{~b})$. But the differences did not reach statistical significance $(\mathrm{p}=0.3$; Table 1).

The study of TIMP1 expression in the tunica media showed statistically significant difference between the 2 groups: its low expression predominated in ICA with pathological deformities ( $84 \%$ of cases) and its high expression - in control group ( $82 \%$ of cases; $p=0.0002$; Figure 2c). The content of TIMP2 was nearly the same in both groups - its elevated expression was noted in most samples (78\% in ICA and $73 \%$ in the control group; $p>0.05$; Table 1, Figure 2d).

We also did not discover any correlations between the degree of expression of MMP2, MMP9, TIMP1, TIMP2 and gender and age, neither in group of pathological deformities of ICA, neither in the control group $(p>0.05)$. There were no associations of these markers with types of pathological deformities as well $(\mathrm{p}>0.05)$. 


\subsubsection{The analysis of Correlations of Expression of Gelatinases and Their Inhibitors}

We examined the correlations of expression of gelatinases and their inhibitors. For this purpose the types of their expression were divided in 4 groups:

1) Low MMP2 or -9 expression and low TIMP1 or -2 expression (for example, MMP $2 \downarrow /$ TIMP $1 \downarrow$ );

2) Low MMP2 or -9 expression and high TIMP1 or -2 expression (for example, MMP9 $\downarrow / T I M P 1 \uparrow$ );

3) High MMP2 or -9 expression and low TIMP1 or -2 expression (for example, MMP2 $\uparrow /$ TIMP1 $\downarrow$ );

4) High MMP2 or -9 expression and high TIMP1 or -2 expression (for example, MMP9 $\uparrow / T I M P 1 \uparrow$ ) (Table 2).
The study of the ratio of the expression of MMP2 and TIMP1 revealed the predominance of high MMP2 and low TIMP1 content in ICA with pathological deformities (78\%, 25 out of 32 patients), while in control group the simultaneous high expression of both markers prevailed $(55 \%, 6$ out of 11 cases; $\mathrm{p}<0.001)$. The similar situation was detected for the ratio MMP9/TIMP1: 71\% (23 out of 32) of patients had high expression of MMP9 and low - TIMP1, 82\% (9 out of 11) of patients of the control group had a synchronous distinct expression of both $(\mathrm{p}<0.05$; Table 2$)$.

The analysis of the MMP2/TIMP2 and MMP9/TIMP2 ratios showed the prevalence of synchronous high expression of these markers in both groups (Table 2).

Table 2. Ratios of expression of gelatinases and their inhibitors in internal carotid artery (ICA) with pathological deformities and in control group.

\begin{tabular}{|c|c|c|}
\hline Ratio of expression & ICA with pathological deformities, $n=32$; absolute number (\%) & Control group, $\mathrm{n}=11$; absolute number (\%) \\
\hline \multicolumn{3}{|l|}{ MMP2/TIMP1 } \\
\hline 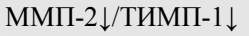 & $4(13)$ & $1(9)$ \\
\hline 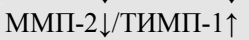 & 0 & $3(27)$ \\
\hline 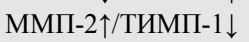 & $25(78)^{*}$ & $1(9)$ \\
\hline 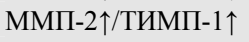 & $3(9)$ & $6(55)$ \\
\hline \multicolumn{3}{|l|}{ MMP9/TIMP1 } \\
\hline 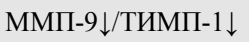 & $4(13)$ & 0 \\
\hline 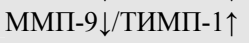 & $1(3)$ & 0 \\
\hline 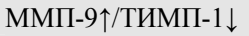 & $23(71)^{*}$ & $2(18)$ \\
\hline 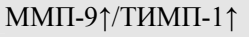 & $4(13)$ & $9(82)$ \\
\hline \multicolumn{3}{|l|}{ MMP2/TIMP2 } \\
\hline 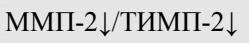 & $3(9)$ & $3(27)$ \\
\hline 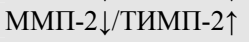 & $1(3)$ & $1(9)$ \\
\hline 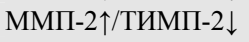 & $4(13)$ & 0 \\
\hline 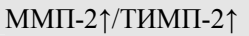 & $24(75)$ & $7(64)$ \\
\hline \multicolumn{3}{|l|}{ MMP9/TIMP2 } \\
\hline 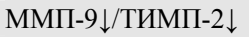 & $3(9)$ & 0 \\
\hline 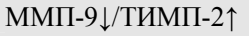 & $2(6)$ & 0 \\
\hline 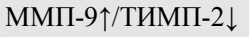 & $4(13)$ & $3(27)$ \\
\hline 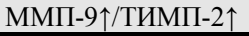 & $23(72)$ & $8(73)$ \\
\hline
\end{tabular}

MMP2: matrix metalloproteinase 2, MMP9: matrix metalloproteinase 9, TIMP1: tissue inhibitor of matrix metalloproteinases type 1, TIMP2: tissue inhibitor of matrix metalloproteinases type $2,{ }^{*}-\mathrm{p}<0.05$

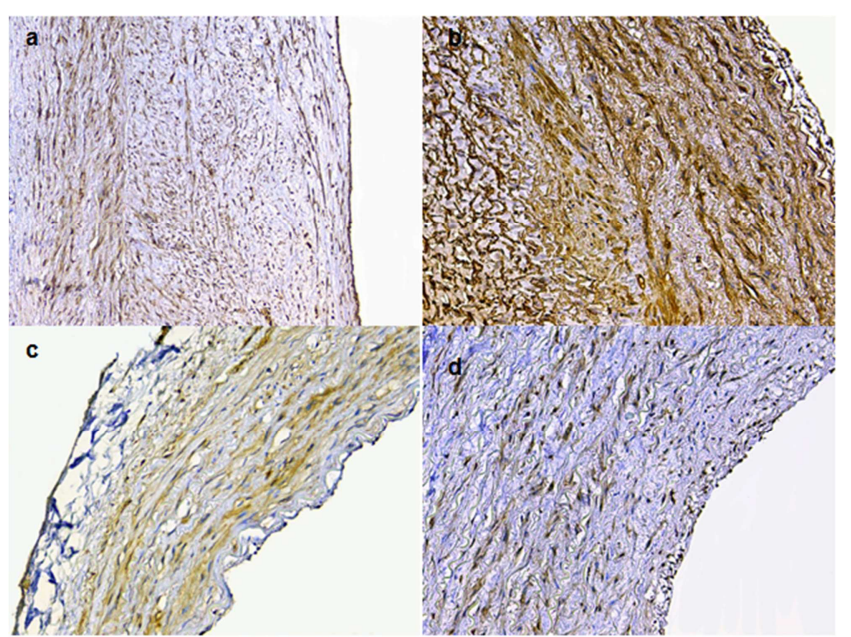

Figure 2. Expression of gelatinases and their inhibitors evaluated by immunohistochemistry. Elevated expression of matrix metalloproteinase 2 (MMP2) (a) (x100 magnification) and MMP9 (b) (x200 magnification) in ICA with fibromuscular dysplasia. High expression of tissue inhibitor of matrix metalloproteinases 1 (TIMP1) in normal artery (c) and TIMP2 - in ICA with fibromuscular dysplasia (x200 magnification).

\subsection{Confocal Laser Scanning Microscopy}

In the study by confocal microscopy of the levels and ratios of expression of MMP-9 and elastin the following values were received. In the control group the expression of MMP-9 was $4.3+0.69 \%$; elastin $16.2+1.48 \%$ (Figure $3 \mathrm{a}$ ). In the group of ICA with a pathological deformities expression of MMP-9 was $26+1.26 \%$; elastin $9.1+0.36 \%$ (Figure $3 b$ ). The significant differences in the expression of MMP9 and elastin (MMP9, $\mathrm{p}<0.03$; elastin, $\mathrm{p}<0.05$ ) have been found when compared between the groups. The co-expression of these markers is noted in $16.9 \%$ of cases (Pearson coefficient $r=0.6$ ). The expression level of TIMP-1 in the control group was $18.4+0.57 \%$ (Figure 3c), and for the ICA group its level was $10.2+1.21 \%$ (Figure $3 \mathrm{~d}$ ). Also the significant differences in expression of MMP9 and TIMP-1 (MMP9, p<0.03; TIMP-1, $\mathrm{p}<0.05$ ) have been found between the control group and ICA group. The co-expression of these markers was detected in $80 \%$ of cases (Pearson coefficient $\mathrm{r}=0.8$ ). 

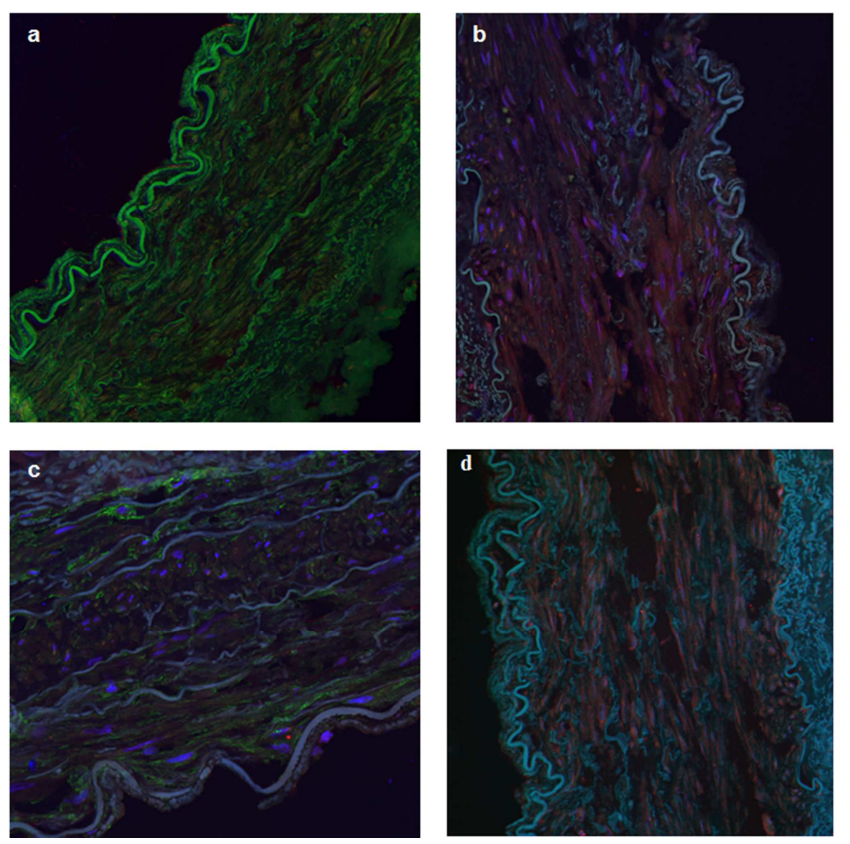

Figure 3. Expression of matrix metalloproteinase 9 (MMP-9), elastin, tissue inhibitor of matrix metalloproteinases (TIMP-1) in internal carotid artery (ICA) with pathological deformities $(b, d)$ and in the control group $(a, c)$ evaluated by confocal laser scanning microscopy (x200 magnification). (a) MMP9 - red fluorescence; elastin - green fluorescence; (c) MMP9 - red fluorescence; TIMP1 - green fluorescence; (b) MMP9 - red fluorescence; elastin - green fluorescence; (d) MMP9 - red fluorescence; TIMP1 - green fluorescence.

Thus, in the control group a high level of the expression of elastin and a low level of the expression of MMP9 have been shown that correlated with the level of TIMP-1. In ICA with pathological deformities group the decrease of the expression of elastin with high activity of MMP9 correlated with low expression of TIMP-1 were registered.

\section{Discussion}

In this study, we conducted the research on the structure of the ICA wall with pathological deformities and some mechanisms of pathogenesis of fibromuscular dysplasia underlying the pathological deformities of ICA with the use of methods of immunohistochemistry and confocal laser scanning microscopy. Our results are consistent with the data of other authors concerning the fragmentation of elastic fibers leading to the violation of elastic properties of the artery. ${ }^{13,15}$ We demonstrate that the expression of collagens I and III remains unchanged in tunica media and adventitia. The reduction of smooth muscle cells confirmed by their marker SMA expression decrease may lead not only to the deterioration of mechanical properties of the artery but also to the disturbance of synthesis of ECM components. The diminution of elastic fibers and smooth muscle cells is shown also by La Barbera G. et al., 2006. ${ }^{14}$

In the current study to our knowledge for the first time we show with the use of immunohistochemistry and confocal laser scanning microscopy the augmented expression of MMPs degrading elastin - MMP2 and MMP9 in ICA with fibromuscular dysplasia. At the same time the expression of their inhibitor TIMP1, but not TIMP2, is diminished. While in normal mammary arteries constituting the control group the synchronous pronounced accumulation of gelatinases and their inhibitor TIMP1 is revealed, in ICA with pathological deformities, there is the disturbance of their balance with a prevalence of MMPs over their inhibitor. This results in intensified breakdown of ECM components, in the first place elastic fibers, what is the main feature of fibromuscular dysplasia of ICA underlying their pathological deformities. $^{13,15}$

The data concerning MMPs and their inhibitors in fibromuscular dysplasia of ICA are not presented in the literature, but their role in the pathogenesis of different diseases of the vessels is well known. For example, in the wall of aortic aneurysm smooth muscle cells in media produce the increased amount of MMP1, MMP2 and MMP9. ${ }^{17,}{ }^{18}$ Here with Wilson W.R. et al., 2005 and Lipp C. et al., 2012 have demonstrated the decrease of TIMP1 and TIMP2 in the aneurysms of the abdominal aorta in comparison with normal aorta that leads to the ECM component degradation. ${ }^{19,20}$

The total analysis of all our data demonstrates that the prevailing "phenotype" of ICA wall with fibromuscular dysplasia has the following characteristics: large amount of smooth muscle cells producing high MMP2 and MMP9 but low TIMP1 (41\%, 13 out of 32 patients). Somewhat less frequently occurred the samples with small amount of smooth muscle cells, but similarly elevated production of gelatinases and reduced TIMP1 (22\%, 7 out of 32 patients). While among normal arteries the samples characterized by high expression of all markers predominated $(\mathrm{p}<0.05)$. TIMP2 expression was high along with elevated MMPs expression in most cases (the difference between groups is not statistically reliable). Probably, the performance of TIMP2 with reduced production of TIMP1 is not sufficient for the suppression of the activity of gelatinases degrading elastin. These findings could contribute the new knowledge to the investigation of fibromuscular dysplasia of ICA and also arteries of other vascular pools.

This study revealed for the first time that different disturbances of balance between the ECM components and proteases degrading them are not determined in the formation of various types of pathological deformities of ICA.

There are several potential limitations that may confound the interpretation of our study findings. Our study was conducted on a small number of patients. Further validation in studies using larger amount of samples is needed. The ICA samples were available from the population of patients who received surgical treatment for the hemodynamically significant pathological deformities. But the characteristics of fibromuscular dysplasia of ICA of patients not having significant hemodynamic changes and not undergoing surgery remains unexplored.

\section{Conclusion}

In conclusion, our results show that the main feature of fibromuscular dysplasia underlying the pathological 
deformities of ICA is fragmentation of elastic fibers, which is caused by the disturbance of balance between gelatinases and their inhibitors. As elastin ensures the strength of the artery under tension it may be one of the causes of the formation of pathological deformities. The reduction of smooth muscle cells causes the abnormality of mechanical properties of the vessel by itself.

\section{Acknowledgments}

The study was supported by B.V. Petrovsky Russian Surgery Research Center, Moscow, Russia and D.O. Ott Research Institute of Obstetrics, Gynecology and Reproductology, Saint Petersburg, Russia.

\section{Conflict of Interest}

None declared.

\section{References}

[1] Horev NG, Pathological tortuosity of internal carotid artery and its surgical treatment. Dissertation, Barnaul; 2000.

[2] Bokeria LA, Sukhanov SG, Katkov AI, Pirtskhalaishvili ZK Surgery of pathological tortuosity of brachiocephalic arteries. Perm: Kursiv; 2006.

[3] Illuminati G, Calio FG, Papaspyropoulos V, Montesano G, D'Urso A Revascularization of the internal carotid artery for isolated, stenotic, and symptomatic kinking. Arch Surg. 2003; 138(2): 192-197.

[4] Nikonenko AS, Gubka AV, Masterukhin AN, Gubka VA Estimation of the cerebral hemodynamics in patients with pathological kinking of arteries originated from the aortal arch according to angiography. Klin Khir. 2000; 10:5-7.

[5] Smirnova YuV, Al'myasheva LI Etiology of pathological deformation of internal carotid arteries and pathogenesis of cerebral circulation disturbances in this anomaly (review). Klinicheskaya Nevrologiya. 2012; 1: 33-38.

[6] Khaimovich G Vascular surgery on Khaimovich in 2 volumes. Under edition E. Asher. Moscow: Binom; 2010.

[7] Timina IE, Burtseva EA, Losik IA Modern approach to the complex ultrasound examination of patients with pathological deformation of internal carotid artery. Angiologiya i Sosudistaya Khirurgiya. 2011; 3: 49-57.
[8] Aleksic M, Schütz G, Gerth S, Mulch J Surgical approach to kinking and coiling of the internal carotid artery. J Cardiovasc Surg. 2004; 45(1): 43-48.

[9] Togay-Işikay C, Kim J, Betterman $\mathrm{K}$ et al. Carotid artery tortuosity, kinking, coiling: stroke risk factor, marker, or curiosity? Acta Neurol Belg. 2005; 105(2):68-72.

[10] Weibel J, Fields WS Tortuosity, coiling and kinking of the internal carotid artery. I. Etiology and radiographic anatomy. Neurology. 1965; 15: 7-18.

[11] Weibel J, Fields WS Tortuosity, coiling and kinking of the internal carotid artery. II. Relationship of morphological variation to cerebrovascular insufficiency. Neurology. 1965; 15: 462-468.

[12] Mumoli N, Cei M Asymptomatic carotid kinking. Circ J. 2007; 72: 682-683.

[13] Kuzyk YuI Pathological deformations of carotid arteries: etiology, pathogenesis, clinical and pathomorphological changes. Angiologiya i Sosudistaya Khirurgiya. 2014; 20(3): 123-128.

[14] La Barbera G, La Marca G, Martino A et al. Kinking, coiling, and tortuosity of extracranial internal carotid artery: is it the effect of a metaplasia? Sur Radiol Anat. 2006; 28(6): 573-580.

[15] Minkina SM Morphogenesis of fibro-muscular dysplasia of renal arteries. Dissertation, Moscow; 1980.

[16] Ponticos M, Smith B Extracellular matrix synthesis in vascular disease: hypertension, and atherosclerosis. J Biomed Res. 2014; 28(1): 25-39.

[17] Bonderman D, Gharehbaghi-Schnell E, Wollenek G, Maurer G, Baumgartner $\mathrm{H}$, Lang IM Mechanisms underlying aortic dilatation in congenital aortic valve malformation. Circulation. 1999; 99: 2138-2143.

[18] Goodall S, Porter KE, Bell PR, Thompson MM Enhanced invasive properties exhibited by smooth muscle cells are associated with elevated production of MMP-2 in patients with aortic aneurisms. Eur J Vasc Endovasc Surg. 2002; 24(1): 72-80.

[19] Lipp C, Lohoefer F, Reeps C et al. Expression of a disintegrin and metalloprotease in human abdominal aortic aneurysms. J Vasc Res. 2012; 49: 198-206.

[20] Wilson WR, Schwalbe EC, Jones JL, Bell PR, Thompson MM Matrix metalloproteinase 8 (neutrophil collagenase) in the pathogenesis of abdominal aortic aneurism. Br J Surg. 2005; 92(7): 828-833. 